\title{
Implementing a Patient Safety Team to reduce serious incidents
}

\author{
Louise Dowell
}

\begin{abstract}
The Division were experiencing a high number of serious incidents, and the Team felt that a good safety strategy would improve the quality of care given. Through multidisciplinary engagement they wanted to learn from these, encourage reporting and focus on a fair blame culture. The ultimate aim was to increase incident reporting, decrease serious incidents and improve quality. The key aim of the project was to improve the quality of care for the woman and their babies, we reduced the incidence of serious incidents and increased the incident reporting of less serious incidents, this was based on the theory of the Heinrich Ratio which theorises that for every serious incident there will be 300 less serious / near miss incidents. The Team wanted to ensure that the multidisciplinary team were engaged and felt confident to report incidents, and would receive the appropriate feedback and support. In addition all staff involved in the incident would be involved in the investigation and be at the heart of the decision making. The key measure for improvement was the increase in incident reporting (44\% increase 2011 âdu 2012) and the decrease in serious incidents. The figures support the theory that the increase in minor incidents being reported and managed has reduced the incidence of serious incidents. Staff engagement in the process was paramount, and this was driven by a passion to ensure the woman was at the centre of every decision or safety improvement that was made. Women and their families would be involved in the quality improvement process.
\end{abstract}

\section{Problem}

The Division were experiencing a high number of serious incidents, and the Team felt that a good safety strategy would improve the quality of care given. Through multidisciplinary engagement they wanted to learn from these, encourage reporting and focus on a fair blame culture. The ultimate aim was to increase incident reporting, decrease serious incidents and improve quality.

In the past staff had felt excluded from incident investigation and were apprehensive when completing incident forms for fear of reprisal. To ensure this strategy worked staff had to feel comfortable to report. The key aim of the project was to improve the quality of care for the woman and their babies, we reduced the incidence of serious incidents and increased the incident reporting of less serious incidents, this was based on the theory of the Heinrich Ratio which theorises that for every serious incident there will be 300 less serious / near miss incidents. (1) Because many accidents share common root causes, addressing more commonplace accidents that cause no injuries can prevent accidents that cause injuries.

\section{Background}

A significant number of serious incidents were being reported and the management team made a commitment to reduce these through sustained and visible leadership and deep employee engagement. The initiative started with a weekly meeting to review of all incidents from that week, with feedback from the previous week. Terms of reference for the group were drawn up and included membership from the multidisciplinary team including obstetricians, paediatricians, midwives and anaesthetists. In addition a novel approach has been adopted where anyone no matter what their job role can attend the meeting. There is active involvement of the Consultant Team who are integral to good risk management within the unit.

Feedback is given to staff on an individual basis and a quarterly Lessons Learned Newsletter is published and sent to all staff. To proactively manage risk measures such as risk assessment, staff discussion, mentoring and problem solving have been introduced. All serious incidents are subject to a case review / Root Cause Analysis. These reviews are attended by senior clinical staff (anaesthetists, surgeons, physiotherapists) from outside the maternity service to ensure learning is shared across the organisation. A culture of fair blame is inherent to our ethos. The whole team are involved, no one is excluded. Risk management training is provided to all staff, a programme of multidisciplinary skills drills further enhances the culture of safety throughout the unit.

\section{Baseline measurement}

The numbers of incidents were recorded, based on their severity score (between 1 and 5) 1 being minor and 5 catastrophic. The aim was to increase the minor incidents and decrease the more serious incidents.

The key measure for improvement was the increase in incident reporting (44\% increase 2011 to 2012) and the decrease in serious incidents (see attached report). The figures support the theory that the increase in minor incidents being reported and managed has reduced the incidence of serious incidents.

See supplementary file: ds1615.docx 


\section{Design}

When reviewing the problem it became clear that the number of serious incidents would need to be reduced. In a complex health environment is important that staff feel comfortable to report incidents and are encouraged to learn from these. Systems may be changed and improved. These changes and lessons learned from the minor incidents help to reduce the number of serious incidents. The Team used the therory of the Heinrich ratio to base their principles on. This theorises for every 300 minor / near miss incidents there will be one serious incident. Therefore managing and reporting the minor incidents was extremely important.

\section{Strategy}

The strategic theme that provided direction was from the Senior Management Team who would demonstrate commitment to safety through strong visible and consistent leadership. The provision of high-quality, affordable, health care is an increasingly difficult challenge, and therefore a robust patient safety process is relevant to all health care professionals; the initiative would ensure that safety and quality were the primary concern for the multidisciplinary team.

Staff engagement in the process was paramount, and this was driven by a passion to ensure the woman was at the centre of every decision or safety improvement that was made. Women and their families would be involved in the quality improvement process. The Team wanted to ensure that the multidisciplinary team were engaged and felt confident to report incidents, and would receive the appropriate feedback and support. In addition all staff involved in the incident would be involved in the investigation and be at the heart of the decision making. The management team has a strong clinical focus, and has engaged the correct staff to promote this; the team are passionate about improving the care for the woman and baby. As a Team they felt they wanted to imbed this culture throughout the service, engaging and promoting the importance of quality and safety to the wider team.

One of the key elements of risk management is to feedback lessons learned and areas of good practice to the team, so this became one of our key objectives. Not only are lessons from incidents reviewed but these are linked with those from complaints, litigation and PALs enquiries. Risk management education is provided for all the nurses and midwives and focuses on incident reporting, risk assessment, complaints, claims and litigation. Focussed education sessions are undertaken if specific learning from an incident is identified. The link between these is highlighted and professional conduct, rules and standards are discussed. The whole team have a good understanding of why risk management is important. The Clinical Governance Lead is based on the unit and operates an 'open door' policy (where there is often a queue). Support is provided for all staff that are involved in an incident or complaint.

\section{Results}

The project is ongoing and provisional figures indicate a further increase in incident reporting and decrease in serious incidents. The results will continue to be monitored. The Patient Safety Incident Review Team was reviewed by the Aston Team Performance Inventory in November 2011; this measures the elements, dimensions and components that deliver effective performance at a team and organisational level. This report was positive and gave the team a catalyst to disseminate their work further. The team members reported a good deal of attachment to the team and to their team colleagues, this suggested that they were motivated to remain and promote the team in to the future. See report summary below.

See supplementary file: ds 1681. docx

\section{Lessons and limitations}

The project is sustainable if the team continue to understand the importance of incident reporting. Their involvement in the investigation and in the development of solutions is essential along with feedback to the whole team. The involvement from the woman and their family in incident investigation has made lessons clearer and more focussed. It appears that even in the most serious of incidents they appreciate involvement in the process, their priority and focus from learning is often slightly different to that of the healthcare professional. The Trust Board have highlighted the Women's Incident Team as a beacon of good practice within the Trust. Within the Maternity Service the quarterly Lessons Learned Newsletter is published and individual feedback given. Training campaigns are implemented as necessary. Incidents are discussed at the Trust Committees to ensure that organisational learning takes place and staff from other specialties are invited to case reviews/ RCAs. The incident review process has been implemented in other areas of the Trust and the Quality Managers have been introduced following the successful implementation in the Woman and Children's Division. The Division has been identified as an area of good practice. Patients are informed that case reviews are taking place and families and women have been involved in the development of time lines and instrumental in the implementation of lessons learned from incidents.

\section{Conclusion}

Implementing the Patient Safety Team has had positive results not only in terms of the reduction of serious incidents but in the culture of the staff. The Aston Perfomance Inventory showed a positive result with team members reporting a good deal of attachment to the team and to their team colleagues, this suggested that they were motivated to remain and promote the team in to the future.

Involvement of the patient and their relatives has given a more informative apporoach to realsing the issues during root cause analysis investigations.

The Team recognise that there will always be incidents, however their aim is to provide a safe, high quality service to all their patients. This has enabled an open, learning culture where all incidents and complaints are reviewed and this has improved 
systems and enabled good practice to be shared.

\section{References}

1. Heinrich HW (1931). Industrial accident prevention: a scientific approach. McGraw-Hill.

\section{Declaration of interests}

None declared

\section{Acknowledgements}

June Davies Consultant Obstetrician and Head of Department, Nicola Parry Head of Midwifery, All The Patient Safety Team at Blackpool Teaching Hodspitals NHS Foundation Trust Families Division 